\title{
What Makes Learning Analytics Research Matter
}

\author{
Alyssa Friend Wise, Simon Knight, Xavier Ochoa \\ Editors-in-Chief — Journal of Learning Analytics — jla.editorial@gmail.com
}

\begin{abstract}
The ongoing changes and challenges brought on by the COVID-19 pandemic have exacerbated long-standing inequities in education, leading many to question basic assumptions about how learning can best benefit all students. Thirst for data about learning is at an all-time high, sometimes without commensurate attention to ensuring principles this community has long valued: privacy, transparency, openness, accountability, and fairness. How we navigate this dynamic context is critical for the future of learning analytics. Thinking about the issue through the lens of JLA publications over the last eight years, we highlight the important contributions of "problem-centric" rather than "toolcentric" research. We also value attention (proximal or distal) to the eventual goal of closing the loop, connecting the results of our analyses back to improve the learning from which they were drawn. Finally, we recognize the power of cycles of maturation: using information generated about real-world uses and impacts of a learning analytics tool to guide new iterations of data, analysis, and intervention design. A critical element of context for such work is that the learning problems we identify and choose to work on are never blank slates; they embed societal structures, reflect the influence of past technologies; and have previous enablers, barriers and social mediation acting on them. In that context, we must ask the hard questions: What parts of existing systems is our work challenging? What parts is it reinforcing? Do these effects, intentional or not, align with our values and beliefs? In the end what makes learning analytics matter is our ability to contribute to progress on both immediate and long-standing challenges in learning, not only improving current systems, but also considering alternatives for what is and what could be. This requires including stakeholder voices in tackling important problems of learning with rigorous analytic approaches to promote equitable learning across contexts. This journal provides a central space for the discussion of such issues, acting as a venue for the whole community to share research, practice, data and tools across the learning analytics cycle in pursuit of these goals.
\end{abstract}

\section{Introduction}

We write this editorial at a time of many transitions: in the world, in education, and in this journal. On a global level, the world is still working its way through the multiple economic, political, and health challenges brought on by the COVID-19 pandemic. In education, the abrupt shift to remote online schooling and the ongoing fluctuations in modes and manners of instruction have exacerbated learning inequities at all levels, leading many to question long-held assumptions about where and how learning best takes place to benefit all students. Related to this, thirst for data that offers insight into how this learning happens is at an all-time high, at times without commensurate attention to ensuring the principles of privacy, transparency, openness, accountability, and fairness that this community has long valued. How we navigate this dynamic context is critical for the future of the field.

At the journal, we look to these broader situations that set the context for our learning analytics (LA) work from a place of much more modest local transition: the handover of JLA to a new editorial leadership team. When we took over the helm in 2016-2017, the journal was in a space of exciting possibilities. Its first three years were marked by notable successes as a new venue for publication and dissemination of the highest quality LA research, with the publication of papers from an interdisciplinary community of authors. Now at the close of 2021, JLA's eighth year of publication, the journal has grown in many ways: it is home to over 200 articles organized in 21 special sections as well as four regular sections for Research Papers, Practice Reports, Extended Conference Papers, and Data \& Tool Reports. This represents the efforts of hundreds of authors across many countries, very often working in collaboration with one another (though expanded representation of work from Asia, Africa, the Middle East, and Latin America is still a goal). Perhaps most notably, in 2020 JLA was indexed by Scopus and Web of Science, a recognition of the high scientific quality of research we publish and a key marker of maturity for any journal.

Other examples of JLA's maturity as a research venue can be seen in our increased focus on transparency, rigour, and the ultimate impact on learning. This journal now encourages transparency by embedding it into our processes and templates, including acknowledgement of all funding and any potential conflicts of interest related to a manuscript. JLA is also leading 
efforts to systematize robust and inclusive conceptualizations of rigour in this interdisciplinary field through last year's SoLAR webinar "What Do We Mean by Rigour in Learning Analytics?" and its upcoming follow-up workshops, as well as by initiating a LASI tutorial on writing and publishing a rigorous LA article.

Equally importantly to rigour, JLA is also working to foster more attention to the impact of our research output. All articles now include Notes for Practice (research papers) and Notes for Research (practice papers) as a vehicle for increasing the lines of communication between these complementary strands in our field. A core goal from the inception of JLA was to provide a space to promote both the research and practice of LA in a manner open and accessible to all (Gašević, Mirriahi, Long, \& Dawson, 2014). As the second editorial team, we have continued and extended this commitment, innovating new opportunities for publication, including additional journal sections and early access release of accepted works.

\section{What Makes for a Journal of Learning Analytics Paper?}

As editors, we are often asked how JLA differs from LAK and what makes the journal the right venue for a particular piece of work. In other words, what makes something a good JLA paper? Starting with the first question, one key difference is that journals operate on a timeline that allows for multiple levels of extended dialogue between authors, reviewers, and editors in cycles of evaluation, revision, and reply. In contrast to single-round "in-or-out" decisions necessitated by tight annual conferences schedules, the journal review process allows authors the chance to respond to reviewer questions and concerns with substantive edits that can help clarify, flesh out, and strengthen contributions. It also presents opportunities to reshape arguments in ways that more centrally speak to the current discourse in the field. An additional important way that journal publications differ from conference proceedings is in how published pieces can relate to one another. As Siemens (2014) wrote in JLA's inaugural issue, journals offer "a space where ideas are presented, challenged, verified, and refuted - a space where concepts of significance can grow and be extended by new researchers" (p. 5).

JLA has explicitly fostered such dialogue in the community through innovative special sections, including ones inviting critical commentaries on papers (Learning Analytics and Learning Theory, 2015), ones soliciting reflections on a growing area of work from scholars in a neighbouring field (Networks in Learning Analytics, upcoming in 2022), and even one opening a debate about the core nature of our field (What's the Problem with Learning Analytics?, 2019). In short, JLA offers an explicit space for robust discourse about LA that both reflects and helps shape the trajectory of the field. Looking back over our eight years of publications can thus help us explore the question of what makes for a good JLA paper and how this has evolved over time.

\section{1. "Problem-Centric" vs. "Tool-Centric" Research}

At the founding of this journal, the inaugural editors framed the scope of JLA as relating to the opportunities and challenges "of collecting, analyzing, and reporting data with the specific intent to understand and improve learning" (Gašević et al., 2014, p1). This definition alongside the explicit intent to "provide a space for promoting both research and practice" (p.1) as exemplified in that initial collection of papers, developed a focus on examining and affecting learning processes from the original 2011 definition of LA as "the measurement, collection, analysis and reporting of data about learners and their contexts, for purposes of understanding and optimizing learning and the environments in which it occurs." Common to both is a focus on (newer, often digital forms of) data and how they can be leveraged to improve learning, with implicit reference to using computational tools and technologies. This represents an approach to defining a field that foregrounds the "what" and the "how."

On the face of it, defining a field as being about the study of a particular kind of tool makes sense. After all, the term "learning analytics" does indeed refer to a set of technologies. So, while we generally find this narrower "thing focused" approach inadequate, there is also a sense in which the field is precisely about - or perceived by some stakeholder groups to be about - the instantiations of a class of technologies. As Reeves and Lin (2020) point out, the history of educational technology has focused on "what works" with attention to "things" (in our case, specific algorithms or dashboards, for example), over regard for specific problems (for example, the difficulties of many students in navigating the transition from secondary to higher education). However, we agree with their conclusion that this narrow "thing-oriented" approach is not ideal, since it is subject to the well-established critiques of "technocentrism" (Papert, 1988) and elides the important questions of "why?" and "to what ends?" Instead, they — and we - favour a driving formulation for meaningful work of "what is the problem, how can we solve it, and what new knowledge can be derived from the solution?" (Reeves \& Lin, 2020, p. 1998). In our context, the driving problems are ones of human learning, the class of solutions relates to the generation and manipulation

\footnotetext{
${ }^{1}$ Website of the 1st International Conference on Learning Analytics and Knowledge 2011: Connecting the Technical, Pedagogical, and Social Dimensions of Learning Analytics (LAK '11), https://tekri.athabascau.ca/analytics/about
} 
of data to inform stakeholders about that learning, and the new knowledge relates to the insights into learning that can be generated, the methods for generating and sharing these, and the impacts the use of analytic information can have on the learning process. We highlight that while important technical problems will need to be solved in order to address educational ones, computational advances and improvements to technical infrastructure do not constitute an end unto themselves, but have meaning for LA in the ways they can help us to better understand or support teaching and learning.

While Reeves \& Lin's (2020) formulation still runs the danger of Maslow's (1966) law of the instrument (which, in colloquial terms, reminds us how having a hammer leads you to see every problem as a nail) there is still value in describing LA work in terms of the problem to be addressed or the kinds of problems that could be addressed. For example, what might change if we reframed work on "predictive analytics" as being about "proactively identifying struggling students?" First, we are explicitly asked to consider key definitions carefully (What does it mean to struggle? Is this the best conceptualization to use? Who is doing the identifying? What kinds of students are we talking about?). Second, it prompts recognition that prediction models are not the only way to achieve this goal, enabling comparisons among different analytics technologies mobilized to meet similar educational needs. For example the LISSA dashboards help to assess academic "risk" using only descriptive analytics (Charleer et al., 2017). Third, we can step back to examine the goal itself, making sure it is a salient and pressing one for those engaged in the educational endeavor. In this case, instructors and administrators might point out that their core challenge is not simply about identifying struggling students but supporting them. This may sound like semantics (certainly we need to know which students will need help in order to assist them), but it also points to the insufficiency of technical solutions that provide no means for mobilizing this knowledge to address the underlying educational problem. There is thus a point at which attention towards scaffolding analytics-informed action (e.g., Pardo et al., 2018; Matz et al., 2021) will be more beneficial than fine-tuning the computational accuracy of a classification model (Kitto, Buckingham Shum, \& Gibson, 2018).

Articulating the problem also allows us to ask second-order questions about what kinds of learning problems get addressed and who sets this agenda (Wise, Sarmiento, \& Boothe, 2021). For example, the framing of "identifying and supporting struggling students" implies a situation in which changing the actions of the identified students is the best means to improve a problematic success rate, rather than considering changes to the material or curriculum that might make a course more accessible or inviting to particular groups of students. Similarly, tools for administrators to address attrition, for instructors to help redesign their courses, and for students to better handle their academic workload may all use the same data (and potentially some of the same analytics) to support student success but are completely different from a conceptual point of view. Humancentred approaches to LA (Buckingham Shum, Ferguson, \& Martinez-Maldonado, 2019) and, within them, participatory design methods (e.g., Dollinger, 2018; Prieto Alvarez, Martinez-Maldonado \& Buckingham Shum, 2020), are ways in which the community is trying to bring more diverse voices to influence not only analytics solutions but also problem definitions, with profound effects downstream for how LA have impact as an agent of change.

\subsection{Closing the Loop}

While defining the problem to be addressed is critical in setting the stage for LA work, perhaps one of the most important factors that differentiates LA from other similar fields of educational research, such as Educational Data Mining (EDM), is the intent to use data traces from learning contexts to improve the same learning processes from which they were collected. This was described elegantly by Doug Clow (2012) as "closing-the-loop," with Wise (2019) further elaborating how such work differs from traditional educational research in that the changes are short-cycle (responses to what is learned from data happen over minutes, hours, or days rather than months or years), direct (data collected from students benefits these same students), and local (changes respond to specific situations rather than generalized ones). This, in many ways, is similar to ideas underlying work on artificial intelligence in education (AIED), with a key distinction being the greater emphasis in LA on human agency or "actionable intelligence" rathwelver than machine-led adaptivity. Is this theoretically distinguishing feature of LA research, however, being realized in practice? Just a few years ago, Ferguson and Clow (2017) stated that "not enough published work is making it clear how the move will be made from researching the data to optimising the learning" (p. 62).

We have seen this in submissions to the journal as well. A common shortcoming of manuscripts is insufficient attention to how the LA research closes, or intends to close the loop. To be clear, the field values, and needs to continue, basic research that advances our ability to generate relevant data traces of learning and model them in useful ways. As noted in the prior section, however, the articulation of the educational context motivating our work holds significant value and practical implications. Therefore, we ask the members of this community to consider how their work might look different by keeping a view towards closing the loop within sight. This can take many different forms. Some of the more proximal ones include designing a tool, building a tool, studying how intended users respond to a tool, examining how people actually use the tool in an authentic learning context, and documenting any effects the tool has on the desired learning. But a view towards closing the loop can also be more distal. For example, when developing new data collection methods or analytic techniques, it could take 
the form of considering potential audiences for the work (teachers, students, designers, or other educational stakeholders) and using that context to inform the technical work.

Put together, we can imagine a prototypical lifecycle for LA work that begins with an important problem in education for which some insight can be generated through the development of new data, metrics, or models. These then form the basis for creating and testing a tool to provide actionable information or feedback based on the data to some set of relevant people. The next stage of work focuses on integrating the tool into real-world educational contexts and understanding the factors needed for success. In turn, this generates information about reception, use, and impact that can inform redesign, or in some cases problem redefinition. A specific manuscript is likely to focus on only one of these stages of work, but in each, an eye towards the next step (as well as grounding in prior ones) can be valuable. For example, when developing new kinds of measurements, consider what kinds of tools for what kinds of users might they eventually be part of. When building and testing a tool, consider how it will be introduced and supported in real-world contexts. When designing how to introduce and support a tool, consider what eventual measures of impact are most valued. Finally, when measures of reception, use, and impact are being determined, consider how they can inform refinement, redesign, or redefinition in a new cycle of work.

\subsection{Continuing the Learning Analytics Cycle}

The lifecycle described above represents LA work as an iterative cycle where the results of evaluating a tool (information generated about its real-world uses and impacts) are used to guide a new cycle of improvement to data, analysis, and intervention design. Recurrent improvement cycles ensure that LA interventions get more effective and impactful with time. With some noteworthy exceptions, often developed and deployed by large groups with support from their institutions, it is relatively rare for LA research to focus on refining existing solutions (Ferguson \& Clow, 2017). This may in part be related to the challenges of extending the development of tools or technical infrastructures developed by others to address shared learning challenges, reiterating the importance of the value of the openness this community has long expressed.

The focus on new ideas and new solutions could be the result of different factors: pressure to publish groundbreaking novel research, attractiveness of new technologies or problems, loss of interest by existing stakeholders, length of typical funding cycles, and so on. No matter the cause, the continual search for new solutions can have a negative impact on the external perception of LA as 1) interesting proof-of-concepts that never make it out of the lab (e.g., advanced predictive models that are built and evaluated, but never deployed at scale for impact ${ }^{2}$ ) or 2) tools bolted onto existing systems without meaningfully drawing on existing research or a deep analysis of practical need (e.g., some usage dashboards in LMSs).

As a community, we need to create structures and incentive systems to promote the cyclical maturation of our solutions. For example, we might imagine opening a new section of the journal specifically dedicated to evaluation and improvement, spotlighting impactful LA rollouts, or a yearly award for the best research-to-practice transition. We should also consider ways to further support openness (in data sets, algorithms, tools, and more) to create a regular practice to build on, refine, and expand the prior work of others. Only by closing the data loop, bringing analytic information back to relevant stakeholders, and then iterating the LA cycle from problem, through design, rollout, and evaluation, leading to improved design, will we achieve the kinds of impact in the education world that make LA matter.

\section{Problems, Solutions, and Impact in the JLA Papers of 2021}

This year we published two special sections on Collaboration Analytics (Schneider, Dowell, \& Thompson, 2021) and Learning Analytics for Primary and Secondary Schools (Kovanović, Mazziotti, \& Lodge, 2021), as well as twelve individual papers in our Research and Extended Conference Papers sections. Across these we can draw out a representation of how the field is moving forward with the issues of articulating problems, closing the loop, and examining impact to continue the LA development cycle.

For example, this year's articles mobilize LA to address several problems, including the lack of learning reflection among adolescents (Cloude et al., 2021), a need for students to read and respond effectively to different collaborative situations (Worsley et al., 2021), and the ways that confusion can impede students' metacognition (Zhang et al., 2021). We also see increased interest in tackling the challenges of more open-ended (Emara et al., 2021) and inquiry-based learning environments (Dickler et al., 2021; Rodríguez-Triana et al., 2021). Part of "closing the loop" continues to be methodological innovation and conceptual development. These contributions are reflected strongly throughout the Collaboration Analytics special section, for example, with three papers focusing on different aspects of modelling collaboration (Halpin, 2021; Swiecki, 2021; Lämsä et

\footnotetext{
${ }^{2}$ Note that while scale, in a simple sense, might be considered as the "size of population" a tool is made available to or used by, we must go beyond to think about scale in terms of the "size of effect" (Knight, Gibson, \& Shibani, 2020). The latter encourages us to consider which $\mathrm{N}$ of users we might have the most positive impact on (rather than simply how to reach the largest $\mathrm{N}$ ).
} 
al., 2021) and two papers advancing theory in the conceptualization of collaboration analytics (Martinez-Maldonado et al., 2021; Han, Krieger, \& Greiff, 2021).

We also see attention to the implementation and use of LA in practice, including examinations of how instructors, tutors, and coaches use dashboards and other tools (van Leeuwen et al., 2021; Olney et al., 2021; Campos et al., 2021; Tsai, WhitelockWainwright, \& Gašević, 2021). Taking a different perspective on implementation, we have also seen papers investigating hands-on issues of working with learning data. These include a Practical Report in one of the Special Sections unpacking the potential of particular kinds of analytic approaches for primary and secondary schools from a system designer perspective (Pelanek, 2021), and an analysis of the potential to re-identify de-identified data via publicly available school data (Yacobson et al., 2021). This latter concern, of course, threads through the community's long-standing attention to ethical and privacy concerns, represented in a review on the topic published this year (Cerratto Pargman, and McGrath (2021). As Krumm, Boyce, and Everson (2021) explain in their paper on bringing together data from instruction- and assessment-focused technologies, practical concerns around data exchange involve both collaboration between systems and people, highlighting the sociotechnical nature of our work. This recognition is central to research that makes visible the range of potentials for the careful design of analytics that are aligned with learning environments to be pedagogically grounded and practically usable in an authentic context (Rodríguez-Triana et al., 2021). Similarly, recognizing the importance of the capacity to use feedback from systems, two further Extended Conference Papers discuss engagement with feedback (Iraj et al., 2021) and student capacity to assess the quality of work (their evaluative judgement; Khosravi et al., 2021).

Another common problem in LA is how to help educators identify students in need of formative feedback. Predictive modelling represents a class of algorithmic approaches often employed as a starting point. As described earlier, such models have the potential to be useful in addressing the challenge of proactively identifying struggling students (among other possible framings of this much-researched problem area). However, the focus on developing these predictive models in LA is not simply improved accuracy as measured by a technical metric such as precision or F1 score, but on their usefulness in driving successful interventions. Works that only compare the technical performance of different modelling methods without critical attention to the larger context of learning are not currently accepted for publication in JLA. As an example of productive uses of predictive modelling, this year we have published Khosravi et al. (2021), an Extended Conference Paper that productively explores the use of human-in-the-loop AI methods to help guide instructors in using predictive models effectively to support students in need. Turning to questions of implementation and impact, Sense, Velde, and Rijn (2021) measure and model the effect of using a prediction-based adaptive fact-learning tool, while Harrison et al. (2021) look at the long-term impact of prediction-based early-warning systems on student retention.

While the journal has published a small number of Practical Reports, establishing a steady stream of submissions to this section of the journal remains a challenge. Such submissions are important for establishing ongoing dialogue between research and practice contexts, as well as among stakeholders. At a basic level, practical reports provide an important outlet for concrete examples of LA implementation in authentic environments, and an opportunity for a wider range of participation in the journal. Moreover, the section instantiates important components of "closing-the-loop" related to impact; for example, by sharing lessons learned in developing or implementing a learning analytic tool to meet a local need, examining the application of research findings to particular problems of practice, or setting out the constraints and challenges of a learning context in which analytics are to be applied. While a growing number of research submissions reflect an increased attention to matters of implementation, and the role of stakeholders across areas - including IT divisions, university instructors, $\mathrm{K}-12$ teachers, students, and those in teaching and learning support roles - we still aim to bring those voices, alongside others, more directly into the journal. This reflects a desire for LA research to be with and, at times, led by practitioners.

We also solicit additional submissions to our Data and Tools section, intended to foster open science through sharing methodological advances in tools and platforms, and datasets that can be used to develop, test, and compare different analytical approaches. As described above, openness is a key prerequisite not only for deeply interrogating the quality of our data and rigour in the way they are analyzed, but also for promoting the collective advancement of LA knowledge and tools rather than simply having gains in siloed efforts.

\section{Conclusion: What Makes Learning Analytics Research Matter}

As we reflect on the development of JLA over the last eight years, we see the metaphor of closing the loop reflected across at least three levels. First, within individual LA projects or tools, closing the loop refers to the provision of outcomes from our analytic approaches back into the learning environments from which they were derived. Second, beyond individual projects, we seek to continue the LA cycle across the field as a collective body of work in which multiple projects build on each other over time, bridging research and practice. Finally, we seek to close the loop between our community spaces and the world we live in, considering how the ways we work together (our processes, practices, structures, norms, and for the journal in 
particular, our editorial policies) animate our values. Our actions as researchers and practitioners exist within a wider educational and societal context, and the impacts we achieve, and aim to achieve, must be seen in this context. Practically, then, this third level of loop closing must recognize that our work takes place not in some abstracted vacuum but within existing systems with their own histories. The learning problems we choose to work on are never blank slates. Rather, they embed societal structures, reflect the influence of past technologies and their social mediation, and have previous enablers, barriers, and attempted solutions acting on them. It is into this complex and profoundly human context that our work eventually acts.

This recognition requires us to ask hard questions: What parts of existing systems, educational and otherwise, is our work challenging? What parts is it reinforcing? Do these effects, intentional or not, align with our values and beliefs? Particular attention should be paid to the language we develop or adopt. For example, what do we commit to by developing analytics of "learning loss"? Considering this question is not just an abstract conceptual exercise; responses must recognize the historical comparisons made between students from different demographic groups and the practical impact of decisions taken based on these comparisons. Metrics and measures matter too. For example, what do we commit to through the acceptance of assessment structures that by their nature motivate the development of remote proctoring analytics? This points us back to the importance of a problem-centric rather than thing-centric conceptualization of LA, placing in sharp relief the value-laden nature of our decisions in choosing what problems to work on and the importance of engaging with diverse educational communities in making these decisions.

At this critical moment, when assumptions, practices, and norms of education have been upended, what do we choose to work on and what is the path we want to chart forward? Do we seek to develop tools that help us re-create pre-pandemic classrooms in new forms? Do we see opportunities for progressive improvement along particular dimensions where data and computation offer inroads to long intransient challenges? Is there an opportunity for analytics to help us fundamentally rethink and refashion our educational systems in powerful ways? (Ladson-Billings, 2021).

To return to the title of this editorial: "What makes learning analytics matter?" The answer lies in our ability to contribute to progress on both immediate and long-standing challenges in learning, not only improving current systems, but also considering alternatives for what is and what could be. This means tackling important problems of learning with rigorous analytic approaches to promote equitable learning across contexts in ways that include stakeholder voices. The value of our field then lies in the ways we navigate (and develop methods to navigate) understanding of learning challenges in their messy real-world contexts, the ways we create, implement, and evaluate tools to address these challenges, and the ways we use this knowledge - whether positive stories of addressing challenges, or unsuccessful or negative impacts — to feed into our ongoing cycles of work.

This journal is a central space for this discussion, acting as a venue for the whole community to share research, practice, data, and tools across the LA cycle, with attention to rigour and an eye towards impact. As its outgoing editors, we are grateful to the community for engaging thoughtfully and critically to navigate these issues. In our stewardship of the journal over the last five years, we have sought to draw on and reflect that collective expertise as we developed new strategies. This work could not have happened without the significant support of JLA's numerous authors, reviewers, guest editors, and editorial board members, whose intellectual contributions are the lifeblood of the journal. We are also eternally grateful for the support of an excellent in-house team. Sameen Reza, Ruth Bradley-St-Cyr, Julia Cochrane, and Rosa Lisa Iannone, we could not do this important work without you. Finally, we want to acknowledge the critical financial support of SoLAR, which provides open access to all publications with no author fees. We are delighted to begin the process of handing over the reins to the incoming editors and we look forward to the ways in which the journal will further mature under their significant expertise.

\section{Declaration of Conflicting Interest}

The authors declared no potential conflicts of interest with respect to the research, authorship, and/or publication of this article.

\section{Funding}

The authors declared no financial support for the research, authorship, and/or publication of this article.

\section{References}

Buckingham Shum, S., Ferguson, R., \& Martinez-Maldonado, R. (2019). Human-centred learning analytics. Journal of Learning Analytics, 6(2), 1-9. https://doi.org/10.18608/jla.2019.62.1

Charleer, S., Moere, A. V., Klerkx, J., Verbert, K., \& De Laet, T. (2017). Learning analytics dashboards to support adviserstudent dialogue. IEEE Transactions on Learning Technologies, 11(3), 389-399. https://doi.org/10.1109/TLT.2017.2720670 
Clow, D. (2012). The learning analytics cycle: Closing the loop effectively. Proceedings of the 2nd International Conference on Learning Analytics and Knowledge (LAK'12), 29 April - 2 May 2012, Vancouver, BC, Canada (pp. 134-138). New York: ACM. https://doi.org/10.1145/2330601.2330636

Dollinger, M. (2018). Technology for the scalability of co-creation with students. In M. Campbell, J. Willems, C. Adachi, D. Blake, I. Doherty, S. Krishnan, S. Macfarlane, L. Ngo, M. O’Donnell, S. Palmer, L. Riddell, I. Story, H. Suri, ... J. Tai (Eds.), Open Oceans: Learning Without Borders. Proceedings of the $35^{\text {th }}$ Annual Conference of the Australasian Society for Computers in Learning in Tertiary Education (ASCILITE 2018), 25-28 November 2018, Geelong, Victoria, Australia (pp. 346-350). Australasian Society for Computers in Learning in Tertiary Education. https://researchrepository.murdoch.edu.au/id/eprint/59155/1/ASCILITE-2018-Proceedings-Final.pdf\#page=348

Ferguson, R., \& Clow, D. (2017). Where is the Evidence?: A Call to Action for Learning Analytics. Proceedings of the Seventh International Learning Analytics \& Knowledge Conference (LAK '17), 13-17 March 2017, Vancouver, BC, Canada (pp. 56-65). New York: ACM. https://doi.org/10.1145/3027385.3027396

Gašević, D., Mirriahi, N., Long, P. D., \& Dawson, S. (2014). Editorial: Inaugural issue. Journal of Learning Analytics, 1(1), 1-2. https://doi.org/10.18608/jla.2014.11.1

Kitto, K., Buckingham Shum, S., \& Gibson, A. (2018). Embracing imperfection in learning analytics. Proceedings of the $8^{\text {th }}$ International Conference on Learning Analytics and Knowledge (LAK '18), 5-9 March 2018, Sydney, NSW, Australia (pp. 451-460). New York: ACM. https://doi.org/10.1145/3170358.3170413

Knight, S., Gibson, A., \& Shibani, A. (2020). Implementing learning analytics for learning impact: Taking tools to task. Internet and Higher Education, 45. https://doi.org/10.1016/j.iheduc.2020.100729

Ladson-Billings, G. (2021). I'm here for the hard re-set: Post pandemic pedagogy to preserve our culture. Equity \& Excellence in Education, 54(1), 68-78. https://doi.org/10.1080/10665684.2020.1863883

Maslow, A. H. (1966). The psychology of science: A reconnaissance. New York: Harper Collins.

Matz, R., Schulz, K., Hanley, E., Derry, H., Hayward, B., Koester, B., et al. (2021). Analyzing the efficacy of ECoach in supporting gateway course success through tailored support. Proceedings of the $11^{\text {th }}$ International Conference on Learning Analytics and Knowledge (LAK '21), 12-16 April 2021, Irvine, CA, USA (pp. 216-225). New York: ACM. https://doi.org/10.1145/3448139.3448160

Papert, S. (1988). A critique of technocentrism in thinking about the school of the future. In B. Sendov \& I. Stanchev (Eds.) Children in the information age (pp. 3-18). Oxford, UK: Pergamon.

Pardo, A., Bartimote, K., Buckingham Shum, S., Dawson, S., Gao, J., Gašević, D., et al. (2018). OnTask: Delivering datainformed, personalized learning support actions. Journal of Learning Analytics, 5(3), $235-249$. https://doi.org/10.18608/jla.2018.53.15

Prieto Alvarez, C., Martinez-Maldonado, R., \& Buckingham Shum, S. (2020, March). LA-DECK: A card-based learning analytics co-design tool. Proceedings of the $10^{\text {th }}$ International Conference on Learning Analytics and Knowledge (LAK '20), 23-27 March 2020, Frankfurt, Germany (pp. 63-72). New York: ACM. https://doi.org/10.1145/3375462.3375476

Reeves, T. C., \& Lin, L. (2020). The research we have is not the research we need. Educational Technology Research and Development, 68(4), 1991-2001. https://doi.org/10.1007/s11423-020-09811-3

Siemens, G. (2014). The Journal of Learning Analytics: Supporting and promoting learning analytics research. Journal of Learning Analytics, 1(1), 3-5. https://doi.org/10.18608/jla.2014.11.2

Wise, A. F. (2019). Learning analytics: Using data-informed decision-making to improve teaching and learning. In O. Adesope \& A. G. Rudd (Eds.), Contemporary technologies in education: Maximizing student engagement, motivation, and learning (pp. 119-143). New York: Palgrave Macmillan. https://doi.org/10.1007/978-3-319-89680-9 7

Wise, A. F., Sarmiento, J. P., \& Boothe, M. (2021). Subversive learning analytics. Proceedings of the 11 ${ }^{\text {th }}$ International Conference on Learning Analytics and Knowledge (LAK '21), 12-16 April 2021, Irvine, CA, USA (pp. 639-645). New York: ACM. https://doi.org/10.1145/3448139.3448210

\section{JLA Papers in 2021}

Campos, F. C., Ahn, J., DiGiacomo, D. K., Nguyen, H., \& Hays, M. (2021). Making Sense of Sensemaking: Understanding How K-12 Teachers and Coaches React to Visual Analytics. Journal of L(3)earning Analytics, 8(3), 60-80. https://doi.org/10.18608/jla.2021.7113

Cerratto, T.P., \& McGrath, C. (2021). Mapping the Ethics of Learning Analytics in Higher Education: A Systematic Literature Review of Empirical Research. Journal of Learning Analytics, 8(2), 123-139. https://doi.org/10.18608/jla.2021.1

Cloude, E., Carpenter, D., Dever, D. A., Lester, J., \& Azevedo, R. (2021). Game-Based Learning Analytics for Supporting Adolescents' Reflection. Journal of Learning Analytics, 8(2), 51-72. https://doi.org/10.18608/jla.2021.7371

Dickler, R., Gobert, J., \& Sao Pedro, M. (2021). Using Innovative Methods to Explore the Potential of an Alerting Dashboard for Science Inquiry. Journal of Learning Analytics, 8(2), 105-122. https://doi.org/10.18608/jla.2021.7153 
Emara, M., Hutchins, N., Grover, S., Snyder, C., \& Biswas, G. (2021). Examining Student Regulation of Collaborative, Computational, Problem-Solving Processes in Open-Ended Learning Environments. Journal of Learning Analytics, 8(1), 49-74. https://doi.org/10.18608/jla.2021.7230

Halpin, P. (2021). Measuring Dynamical Interdependence in Small-Group Collaborations. Journal of Learning Analytics, 8(1), 95-112. https://doi.org/10.18608/jla.2021.7241

Han, A., Krieger, F., \& Greiff, S. (2021). Collaboration Analytics Need More Comprehensive Models and Methods: An Opinion Paper. Journal of Learning Analytics, 8(1), 13-29. https://doi.org/10.18608/jla.2021.7288

Harrison, S., Villano, R., Lynch, G., \& Chen, G. (2021). Microeconometric Approaches in Exploring the Relationships Between Early Alert Systems and Student Retention: A Case Study of a Regionally Based University in Australia. Journal of Learning Analytics, 8(3), 170-186. https://doi.org/10.18608/jla.2021.7087

Iraj, H., Fudge, A., Khan, H., Faulkner, M., Pardo, A., \& Kovanović, V. (2021). Narrowing the Feedback Gap: Examining Student Engagement with Personalized and Actionable Feedback Messages. Journal of Learning Analytics, 8(3), 101116. https://doi.org/10.18608/jla.2021.7184

Khosravi, H., Gyamfi, G., Hanna, B. E., Lodge, J., \& Abdi, S. (2021a). Bridging the Gap Between Theory and Empirical Research in Evaluative Judgment. Journal of Learning Analytics, 8(3), 117-132. https://doi.org/10.18608/jla.2021.7206

Khosravi, H., Shabaninejad, S., Bakharia, A., Sadiq, S., Indulska, M., \& Gašević, D. (2021). Intelligent Learning Analytics Dashboards: Automated Drill-Down Recommendations to Support Teacher Data Exploration. Journal of Learning Analytics, 8(3), 133-154. https://doi.org/10.18608/jla.2021.7279

Kovanovic, V., Mazziotti, C., \& Lodge, J. (2021b). Learning Analytics for Primary and Secondary Schools. Journal of Learning Analytics, 8(2), 1-5. https://doi.org/10.18608/jla.2021.7543

Krumm, A. E., Boyce, J., \& Everson, H. T. (2021). A Collaborative Approach to Sharing Learner Event Data. Journal of Learning Analytics, 8(2), 73-82. https://doi.org/10.18608/ila.2021.7375

Lämsä, J., Uribe, P., Jiménez, A., Caballero, D., Hämäläinen, R., \& Araya, R. (2021). Deep Networks for Collaboration Analytics: Promoting Automatic Analysis of Face-to-Face Interaction in the Context of Inquiry-Based Learning. Journal of Learning Analytics, 8(1), 113-125. https://doi.org/10.18608/jla.2021.7118

Martinez-Maldonado, R., Gašević, D., Echeverria, V., Fernandez Nieto, G., Swiecki, Z., \& Buckingham Shum, S. (2021). What Do You Mean by Collaboration Analytics? A Conceptual Model. Journal of Learning Analytics, 8(1), $126-153$. https://doi.org/10.18608/jla.2021.7227

Olney, T., Walker, S., Wood, C., \& Clarke, A. . (2021). Are We Living In LA (P)LA Land? Reporting on the Practice of 30 STEM Tutors in their Use of a Learning Analytics Implementation at the Open University. Journal of Learning Analytics, 8(3), 45-59. https://doi.org/10.18608/jla.2021.7261

Pelanek, R. (2021). Analyzing and Visualizing Learning Data: A System Designer's Perspective. Journal of Learning Analytics, 8(2), 93-104. https://doi.org/10.18608/jla.2021.7345

Rodríguez-Triana, M. J., Prieto, L. P., Dimitriadis, Y., de Jong, T., \& Gillet, D. (2021). ADA for IBL: Lessons Learned in Aligning Learning Design and Analytics for Inquiry-Based Learning Orchestration. Journal of Learning Analytics, 8(2), 22-50. https://doi.org/10.18608/jla.2021.7357

Schneider, B., Dowell, N., \& Thompson, K. (2021). Collaboration Analytics - Current State and Potential Futures. Journal of Learning Analytics, 8(1), 1-12. https://doi.org/10.18608/jla.2021.7447

Sense, F., van der Velde, M., \& van Rijn, H. (2021). Predicting University Students' Exam Performance Using a Model-Based Adaptive Fact-Learning System. Journal of Learning Analytics, 8(3), 155-169. https://doi.org/10.18608/jla.2021.6590

Swiecki, Z. (2021). Measuring the Impact of Interdependence on Individuals During Collaborative Problem-Solving. Journal of Learning Analytics, 8(1), 75-94. https://doi.org/10.18608/jla.2021.7240

Tsai, Y.-S., Whitelock-Wainwright, A., \& Gašević, D. (2021). More Than Figures on Your Laptop: (Dis)trustful Implementation of Learning Analytics. Journal of Learning Analytics, 8(3), 81-100. https://doi.org/10.18608/jla.2021.7379

van Leeuwen, A., Knoop-van Campen, C. A. N., Molenaar, I., \& Rummel, N. (2021). How Teacher Characteristics Relate to How Teachers Use Dashboards: Results From Two Case Studies in K-12. Journal of Learning Analytics, 8(2), 6-21. https://doi.org/10.18608/jla.2021.7325

Worsley, M., Martinez-Maldonado, R., \& D’Angelo, C. (2021). A New Era in Multimodal Learning Analytics: Twelve Core Commitments to Ground and Grow MMLA. Journal of Learning Analytics, 8(3), 10-27. https://doi.org/10.18608/jla.2021.7361

Yacobson, E., Fuhrman, O., Hershkovitz, S., \& Alexandron, G. (2021). De-identification is Insufficient to Protect Student Privacy, or - What Can a Field Trip Reveal?. Journal of Learning Analytics, 8(2), 83-92. https://doi.org/10.18608/jla.2021.7353 
Zhang, Y., Paquette, L., Baker, R. S., Ocumpaugh, J., Bosch, N., Biswas, G., \& Munshi, A. (2021). Can Strategic Behaviour Facilitate Confusion Resolution? The Interplay Between Confusion and Metacognitive Strategies in Betty's Brain. Journal of Learning Analytics, 8(3), 28-44. https://doi.org/10.18608/jla.2021.7161 